patients, with a maximum stimulation index of 52.4 compared to a maximum of 6.75 in the $\mathrm{HC}$ group. Only $1 \mathrm{HC}$ sample responded with an SI greater than 3.0 , whereas $50 \%$ of RA patients elicited responses above this. Two of the RA patients failed to respond to the peptide pool $(S I<1.0)$. Of note, RA patients were not selected on the basis of tissue type whereas selected peptides bind preferentially to class II HLA containing the shared epitope (SE).

Conclusion: In non-HLA typed individuals, cit-peptide induced proliferative $T$ cell responses were detectable in both RA patients and HCs, and although SIs overall were higher amongst RA patients this did not reach statistical significance in this small sample. Not all RA patients responded to the peptide pool which may be due to the limited number of citrullinated epitopes used, or to RA patients with a non-SE HLA type. Additional work should establish the need for HLA typing in this assay; around half of seropositive RA patients would be expected to be SE positive ${ }^{3}$. Furthermore, a wider array of cit-peptides may be needed to demonstrate autoreactivity in a broader cross-section of RA patients. Our future plans are to further phenotype the cellular subsets responding to the peptide pool and to study assay data in the context of clinical outcomes, to assess its utility for immune monitoring.

References:

[1] McInnes, I. B. \& Schett, G. N Engl J Med. 2011; 365(23):2205-2219

[2] James, E. A et al. Arthritis Rheum. 2014; 66(7):1712-1722

[3] Thomson, W et al. Arthritis Rheum. 1999; 42(4):757-762

Disclosure of Interests: Jessica Swift: None declared, James Stanway: None declared, Catharien Hilkens: None declared, Amy Anderson: None declared, Arthur Pratt Grant/research support from: Pfizer, GlaxoSmithKlein, John Isaacs Consultant of: AbbVie, Bristol-Myers Squibb, Eli Lilly, Gilead, Janssen, Merck, Pfizer, Roche

DOI: 10.1136/annrheumdis-2020-eular.4687

\section{OP0130 IN VITRO CHARACTERIZATION OF INFLAMMATORY ARTHRITIS ASSOCIATED WITH IMMUNE CHECK POINT INHIBITION}

A. S. Sørensen ${ }^{1}$, M. Nørgaard Andersen ${ }^{1}$, K. Juul-Madsen ${ }^{1}$, C. Skejø ${ }^{1}$, H. Schmidt ${ }^{2}$, T. Vorup-Jensen ${ }^{1}$, T. W. Kragstrup ${ }^{1,2}$. 'Aarhus University, Aarhus, Denmark; ${ }^{2}$ Aarhus University Hospital, Aarhus, Denmark

Background: During cancer treatment with immune checkpoint inhibitors (ICI) such as the anti-PD-1 antibody pembrolizumab, $2-4 \%$ of patients develop inflammatory arthritis as an immune related adverse event and half of patients with pre-existing inflammatory arthritis have disease flares. This type of adverse events shows striking similarities with traditional immune mediated inflammatory arthritis. However, the underlying immunological mechanisms of inflammatory arthritis associated with $\mathrm{ICl}$ are not fully understood.

Objectives: We aimed to develop an in vitro model of inflammatory arthritis associated with $\mathrm{ICl}$, and to use this model to investigate monocyte differentiation and activation following treatment with pembrolizumab.

Methods: First, synovial fluid mononuclear cells (SFMCs) and peripheral blood mononuclear cells (PBMCs) from patients with immune mediated inflammatory arthritis (rheumatoid arthritis and peripheral spondyloarthritis, $n=22$ ) and PBMCs from healthy controls were incubated with pembrolizumab and assessed for monocyte chemoattractant protein 1 (MCP-1) secretion by ELISA. Then, cytokine production in SFMCs was studied in more detail by the multiplex V-PLEX proinflammatory panel and by intracellular flow cytometry. Finally, pembrolizumab treated SFMCs were incubated with the different disease modifying anti-rheumatic drugs adalimumab, tocilizumab, tofacitinib, and baricitinib.

Results: Pembrolizumab significantly increased MCP-1 production in the SFMCcultures $(P=0.0031)$. In contrast, pembrolizumab did not change MCP-1 production by PBMCs from neither patients nor healthy controls $(P=0.77$ and $P=0.43)$. Pembrolizumab also increased the production of TNFa $(P=0.049)$, IFNY $(P=0.047)$, and IL-12p70 $(P=0.031)$, but did not change the production of IL-6 $(P=0.98)$. Among SFMCs treated with pembrolizumab there was an increased frequency of intermediate monocytes $(P=0.044)$. Interestingly, pembrolizumab also increased the MCP-1 production within the intermediate monocytes only $(P=0.028)$. In contrast, among SFMCs treated with LPS, only the classical monocyte subset was increased $(\mathrm{P}=0.0045)$ and $\mathrm{MCP}-1$ production increased in both intermediate and classical monocyte subsets. Lastly, the TNFa inhibitor adalimumab and the Janus kinase inhibitors baricitinib and tofacitinib attenuated the pembrolizumab-induced MCP-1 production $(\mathrm{P}=0.0004, \mathrm{P}=0.033$, and $\mathrm{P}=0.025$, respectively) while this was not seen with the IL-6 inhibitor tocilizumab $(P=0.75)$. Conclusion: We have developed a very simple in vitro model of inflammatory arthritis associated with $\mathrm{ICl}$. Using this model, we found that pembrolizumab specifically activated intermediate monocytes and induced TNFa, IFN $\gamma$, and IL-12p70 production, whereas IL-6 was unchanged. These findings were supported by effectful reduction of MCP-1 secretion with TNFa inhibition but not with IL-6 inhibition. This model could potentially be used to further study the effects of ICls and the underlying immunological mechanisms of inflammatory arthritis associated with $\mathrm{ICl}$

References: None.

Disclosure of Interests: Anne Sofie Sørensen: None declared, Morten Nørgaard Andersen: None declared, Kristian Juul-Madsen: None declared, Cæcilie Skejø: None declared, Henrik Schmidt: None declared, Thomas Vorup-Jensen: None declared, Tue Wenzel Kragstrup Shareholder of: iBio Tech ApS, Consultant of: Bristol-Myers Squibb, Speakers bureau: TWK has engaged in educational activities talking about immunology in rheumatic diseases receiving speaking fees from Pfizer, Bristol-Myers Squibb, Eli Lilly, Novartis, and UCB.

DOI: 10.1136/annrheumdis-2020-eular.1356

\section{OP0131 GUT DERIVED ACETATE PROMOTES REGULATORY B CELLS WITH ANTI-INFLAMMATORY EFFECTS}

C. Daien ${ }^{1,2,3}$, J. Tan $^{2}$, R. Audo ${ }^{3}$, J. Mielle ${ }^{1}$, L. Macia ${ }^{2} .{ }^{1}$ IGMM UMR5535, Montpellier, France; ${ }^{2}$ Charles Perkins Centre, The University of Sydney, Sydney, Australia; ${ }^{3}$ University and Hospital of Montpellier, Rheumatology, Montpellier, France

Background: Regulatory B cells (Bregs) are defective in many auto-immune dis eases, i.e. rheumatoid arthritis (RA). The short-chain fatty acid (SCFA) acetate derived mostly from gut microbial fermentation of dietary fiber, promotes anti-inflammatory regulatory $T$ cells and protects mice from type 1 diabetes and colitis. We hypothesized that acetate could be a good candidate to promote Bregs in auto-immune diseases.

Objectives: To assess the effect of acetate on Breg number and function, in vitro and in vivo in mice and humans.

Methods: Bregs were defined as IL-10 producing regulatory B cells (B10 cells) Their number was assessed after overnight exposure to acetate (Ac $10 \mathrm{mM}$ ) and 4 hours of $\mathrm{CpG}$, ionomycin and PMA in mice and after 24 hours of acetate +/- CpG and 4 hours of ionomycin and PMA in humans. Acetate was given to mice either intraperitoneally (twice at a 12-hour interval) or in drinking water for 3 weeks. Acetate-treated B cells were transferred to mice with collagen-antibody -induced arthritis to assess their function. To decipher the mechanisms behind the effect of acetate, we used inhibitors of GPR43 (CATPB), ATP synthase (oligomycin), glycolysis (2-DG), ACSS2 and ACLY and assessed protein lysine acetylation by flow cytometry on human $B$ cells. Acetate and $B 10$ cells were also assessed before and after a 7-day high-fibre diet in 12 healthy volunteers.

Results: In mice, acetate promoted B10 cell differentiation both in vitro (medians [IQR] 3.1 [0.4-3.7] and 9.9 [5.9-17.6]\% of B for $\mathrm{CpG}$ and $\mathrm{CpG}+\mathrm{Ac}$ respectively, $\mathrm{p}=0.002)$ and in vivo when intraperitoneal injected (22 [14-29] and 31 [25-37]\% of $B$ for $P B S$ and acetate respectively, $\mathrm{p}=0.03)$ or added to drinking water $(17[6-25]$ and $39[26-40] \%$ of $B$ for water or acetate respectively, $p=0.02$ ). Adoptive transfer of acetate-treated $B$ cells protected mice from arthritis compared to non-exposed $B$ cells (ANOVA $p=0.008$ ). Acetate also promoted $B 10$ cells from human blood cells (2.5 [1.6-2.7] and 3.4 [2.6-4.5] for unstimulated [Un] and Ac respectively, $\mathrm{p}=0.0001$ ). Conversely to $\mathrm{CpG}$, acetate specifically promoted IL-10, with no impact or a decrease of proinflammatory cytokines (IL-6: 17 [5-29]; 12 [3-21] and 40 [20-47]\% B cells for Un, Ac and CpG respectively, $\mathrm{p}<0.01$ for all comparisons and TNF-a: 48 [29-61]; 41 [28-67] and 69 [64-78]\% B cells for Un, Ac and CpG respectively, $\mathrm{p}<0.01$ for $\mathrm{CpG}$ vs Un or Ac, NS for acetate vs Un). Inhibition of GPR43 and ACLY did not impact acetate response, while inhibition of glycoly sis significantly decreased its effect. Blockade of ACSS2, converting acetate into acetyl-CoA, decreased acetate-induced B10 cells. Acetate was associated with an increase of protein lysine acetylation which was not observed in presence of CpG alone, suggesting a different mechanism of action (2.0 [1.3-3.4]; 3.3 [2.4-5.4] and $1.4[0.5-1.7] \%$ B cells for Un, Ac and CpG respectively, $p=0.002$ for Un vs Ac NS with $\mathrm{CpG}$ ). Conversion of acetate into acetyl-CoA could thus be used for the acetylation of cytoplasmic protein, a post-translational modification that regulates key cellular processes, including energy metabolism. In addition, B10 cells had significantly more lysine-acetylated proteins than IL-10 ${ }^{\text {neg }} \mathrm{B}$ cells or $\mathrm{TNF}^{+} \mathrm{B}$ cells (5.3[3.9-7.3]; 3.2 [2.4-5.4] and 3.9 [2.7-6.2] \% of B for B10, IL-10 ${ }^{\text {neg }} B$ cells or $\mathrm{TNF}^{+}$ $B$ cells respectively, $p<0.01$ for all comparisons). Finally, dietary fiber supplementation in healthy individuals was associated with increased acetate and B10 cells in the blood, which were significantly correlated $\left(R^{2}=0.20, p=0.02\right)$.

Conclusion: Our results suggest that acetate induces functional Bregs, through its conversion into acetyl-CoA, used for cell metabolism and protein acetylation. Delivery of acetate or acetate producing diets or bacteria might be a promising approach to restore Bregs in non-communicable diseases such as RA in which they are defective.

Disclosure of Interests: Claire DAIEN Grant/research support from: from Pfizer, Abbvie, Roche-Chugaï, Novartis, Abivax, Sandoz, Consultant of: Abbvie, Abivax BMS, MSD, Roche-Chugaï, Lilly, Novartis, Speakers bureau: Abbvie, Abivax, BMS, MSD, Roche-Chugaï, Lilly, Novartis, Jian Tan: None declared, Rachel Audo: None declared, Julie Mielle: None declared, Laurence Macia: None declared DOI: 10.1136/annrheumdis-2020-eular.4924 